www.jmscr.igmpublication.org

Impact Factor (SJIF): 6.379

Index Copernicus Value: 71.58

ISSN (e)-2347-176x ISSN (p) 2455-0450

crossref DOI: https://dx.doi.org/10.18535/jmscr/v6i5.177

Journal Of Medical Science And Clinical Research

IGM Publication

An official Publication of IGM Publication

\title{
Diagnostic Value of Pre treatment Neutrophil-to-Lymphocyte Ratio, Platelet-to-Lymphocyte Ratio, and Lymphocyte-to-Monocyte Ratio for Invasive Bladder Carcinoma
}

\author{
Authors
}

N. Madhavan, A. T. Rajeevan*, Edikkula Varghese, K. Rajeevan, Manikandan, A. V. Venugopal, Shanmugha Das.K.V, Felix Cardoza

Government Medical College, Kozhikode, Kerala, India

*Corresponding Author

\section{A. T. Rajeevan}

\begin{abstract}
Purpose: Oncologic outcomes in diverse malignancies are associated with inflammation-based prognostic scores including neutrophil-to-lymphocyte ratio (NLR), platelet-to-lymphocyte ratio (PLR), and lymphocyteto-monocyte ratio (LMR). In this study, we evaluated the predictive value of pre treatment prognostic scores in differentiating muscle invasive (MIBC) and non muscle invasive (NMIBC) bladder cancer.

Materials and Methods: This prospective cross sectional study analysed consecutive transurethral resection of bladder tumour (TURBT) cases from September 2016 to December 2017. Demographics of patients, characteristics of tumour and prognostic scores results were recorded. Prognostic score cut offs were determined using receiver operating characteristics curves. The association between variables and MIBC were evaluated by performing univariate and multivariate binomial logistic regression analysis.

Results: Total patients included were 142. Of this 96 were having NMIBC (stage T1) and 46 were having MIBC (stage T2+). Median age was 75 years. 128 patients were male $(90.1 \%)$ and 14 were female $(9.9 \%)$. The NLR had the greatest area under the curve (AUC) of 0.748 (cut off was 3.89), followed by LMR (cutoff $<1.8$; $A U C, 0.644)$ and PLR (cut off $>218$; AUC, 0.596). Univariate analysis identified NLR, PLR, LMR, Tumour size and Tumour multiplicity as significant predictors of muscle-invasive bladder cancer (MIBC) Table: 15. The multivariate logistic regression model identified NLR (OR, 11.822; 95\% CI, $4.492-31.112 ; p=0.001)$ and tumour size (OR, 6.306; 95\% CI, 1.563 - 25.436; $p=0.010)$ as independent predictors of muscle-invasive bladder cancer $(M I B C)$.

Conclusions: NLR may be used as a simple, cost-effective and easily measured marker for MIBC. It can be performed at the time of diagnostic cystoscopy, and can be used in the planning of further treatment.

Keywords: Urinary bladder neoplasms; Blood platelets; Neutrophils; Lymphocytes.
\end{abstract}

\section{Introduction}

Bladder cancer represents the most common malignancy of the urinary tract and ninth most common cancer worldwide ${ }^{[1]}$. Changes in circulating inflammatory cells are seen in cancer, as cancer triggers a systemic response.
Neutrophilia with relative lymphocytopenia or thrombocytosis are the main changes. The cytokines and chemokines, which are produced by these cancer cells, play a role in the growth, maturation and differentiation of cells within the tumour micro environment ${ }^{[2]}$. 
Nearly $75 \%-85 \%$ of patients present with nonmuscle invasive bladder cancer (NMIBC). Transurethral resection of bladder tumour (TURBT) remains the standard first-line treatment for $\mathrm{NMIBC}^{[3]}$. Bladder cancer staging is most accurately performed with TURBT pathology specimens at present with reasonable accuracy, but still with limitations in pathological staging ${ }^{[4]}$. A number of inflammation-based prognostic scores that measure these changes in microenvironment of cancer cells, including preoperative neutrophil-to-lymphocyte ratio (NLR), platelet-to-lymphocyte ratio (PLR), and lymphocyte-to-monocyte ratio (LMR) are described. These have been found to be associated with the oncologic outcomes in a range of diverse malignancies, including renal, colorectal, hepatic, breast and lung ${ }^{[5,6,7,8]}$.

Many studies establishing a relationship between elevated NLR and invasive bladder cancer have been published ${ }^{[9,10,11]}$. Some of these have neglected other, alternate inflammation-based scores including PLR and LMR, which were included in few studies ${ }^{[13]}$. The aim of our study was to evaluate the predictive value of pre treatment inflammation-based prognostic scores for differentiating muscle-invasive and nonmuscle-invasive disease in patients undergoing TURBT for primary bladder cancer

\section{Material and Methods \\ Patients}

Consecutive patients who underwent TURBT for primary transitional cell bladder cancer between September 2016 to December 2017 at Government Medical College, Calicut were studied prospectively. Patients with nontransitional cell bladder carcinoma, metastatic disease, recurrent bladder tumours, evidence of active infection (including urinary tract infection), alternative cancer/haematological disorder diagnosis, or lacking preoperative blood tests were excluded from the study. All patients had undergone cystoscopic evaluation and had negative urinalyses at time of surgery. Diagnosis of bladder cancer was confirmed by histology, and samples were categorised as NMIBC (stage pTa or T1) or MIBC (stage T2+). For quality assurance, all specimens were confirmed to contain detrusor muscle. Patients with specimens lacking detrusor muscle had a repeat TURBT at 6 weeks. Age, sex, preoperative full blood count, tumour size, tumour grade, and multiplicity were the clinicopathologic variables recorded.

\section{Blood analysis}

As part of a preoperative protocol complete blood counts were routinely collected. Samples were collected in ethylenediaminetetraacetic acid anticoagulated tubes and analysed using Sysmex XE-2100 and XE-5000 Haematology Analysers (Sysmex UK, Milton Keynes, UK). Concurrent urine dipstick and blood tests were performed for patients attending preoperative assessment clinic. Positive urine dipstick tests were sent for midstream urine microbiology and culture and antibiotics were prescribed. Repeat urine dipstick and blood tests were performed prior to the operation in these patients. Preoperative full blood counts within 60 days of TURBT were used for analysis. The sample values closest to the date or resection were analysed when multiple values existed for a patient.

\section{Statistical Analysis}

IBM SPSS Statistics ver. 20.0 was used for statistical analysis. NLR was calculated as the absolute neutrophil count divided by the absolute lymphocyte count. PLR was defined as the absolute platelet count divided by the absolute lymphocyte count. LMR was calculated as the absolute lymphocyte count divided by the absolute monocyte count. Receiver operating characteristics (ROC) curves were generated to determine cutoff points for each prognostic score. Patients were stratified into groups based upon the cutoff/threshold points, and characteristics compared using a chi-square test. For the variables identified as statistically significant in univariate analysis, multivariate analysis was performed using logistic regression. 


\section{Result}

Total patients included in this study was 142, of this 96 were with NMIBC and 46 were with MIBC. Majority were male patients (128,ie $90.1 \%$ ) with a median age of 75 years (interquartile range [IQR], 65-81 years). Of the NMIBC patients, 62 had Ta disease and 34 had T1 disease. 61 of $96(63.5 \%)$ NMIBC patients and 28 of $46(60.9 \%)$ MIBC patients were grade G1 of G2 disease. Full blood count samples were taken 12 days prior to TURBT surgery.

ROC analysis and ROC curves for the four inflammation-based prognostic scores, NLR, PLR, and LMR, using MIBC (stage T2 or greater disease) as a classification variable, are displayed in table 1 and Fig. 1.

Table: 1 Table showing ROC analysis

\begin{tabular}{|c|c|c|c|c|c|}
\hline Test variables & $\begin{array}{c}\text { Area under } \\
\text { the curve }\end{array}$ & \multirow{2}{*}{$\mathrm{SE}$} & $\mathrm{p}$ value & \multicolumn{2}{c|}{$95 \%$ Confidence Interval } \\
\cline { 4 - 6 } & 0.748 & 0.047 & $0.001^{*}$ & 0.654 & 0.841 \\
\hline NLR & 0.596 & 0.053 & 0.065 & 0.492 & 0.699 \\
\hline PLR & 0.644 & 0.052 & $0.006^{*}$ & 0.541 & 0.746 \\
\hline LMR & Lp $<0.05$, statistically significant
\end{tabular}

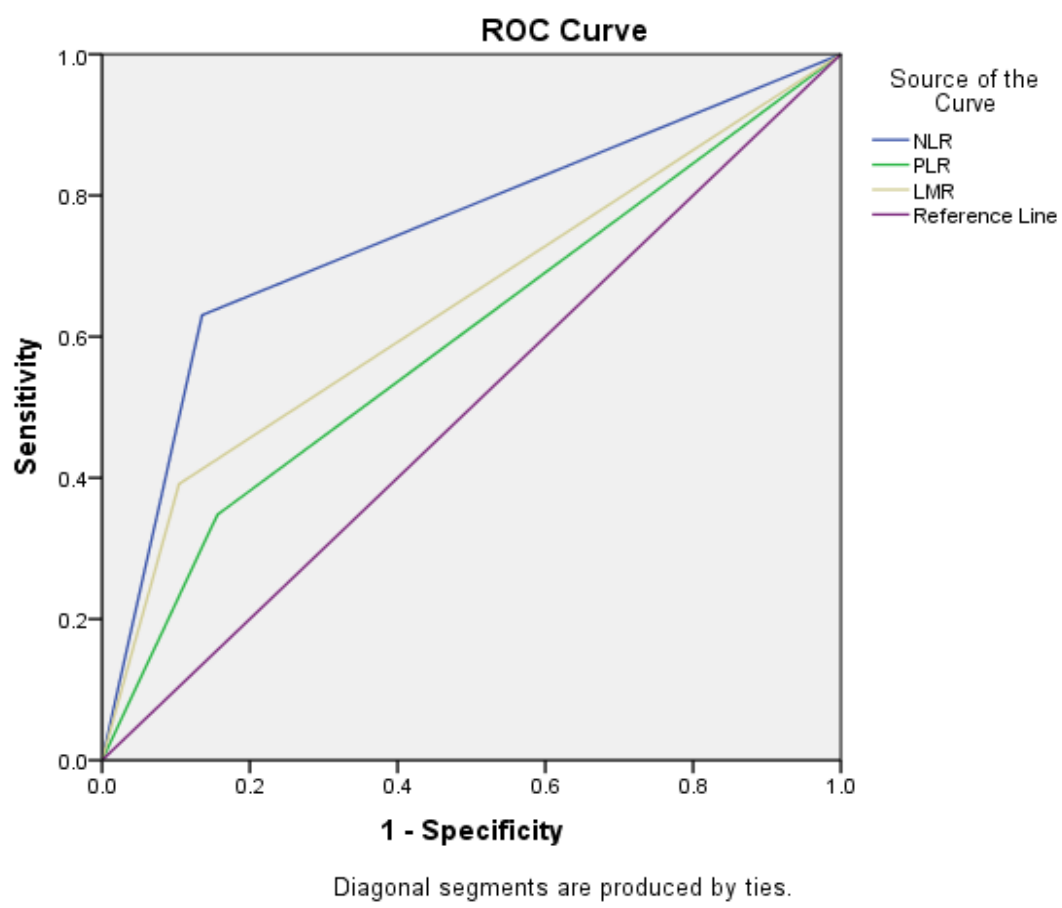

Figure1: Receiver operating characteristics curves for inflammation-based prognostic scores

Of the prognostic scores, NLR had the greatest area under the ROC curve (AUC) of 0.748 (sensitivity, 69.0\%; specificity, 83.0\%; $\mathrm{p}<0.001$ ), followed by LMR area under the ROC curve (AUC) of, 0.644 (sensitivity, 64.3\%; specificity, 75.4\%; $\mathrm{p}=0.006$ ) and PLR area under the ROC curve (AUC) of 0.596 (sensitivity. 51.6\%; specificity. 73.0\%; $\mathrm{p}=0.065$ ).

Based upon the ROC threshold values patients were stratified into groups. Table 2,3,4 and 5 describes he patients' baseline characteristics and comparisons of the patients' clinicopathological characteristics stratified by NLR, PLR, and LMR. Chi-square analysis, with p-values $<0.05$ considered significant, is used to compare groups. 
Table: 2 Baseline characteristics of patients associated with Neutrophil-to-lymphocyte ratio (NLR)

\begin{tabular}{|c|c|c|c|c|c|}
\hline \multirow[t]{2}{*}{ Age } & \multicolumn{2}{|c|}{ NLR } & \multirow[t]{2}{*}{ Total } & \multirow{2}{*}{$\begin{array}{l}\text { Chi square } \\
\text { value }\end{array}$} & \multirow{2}{*}{$\mathrm{p}$ value } \\
\hline & $<3.89$ & $>3.89$ & & & \\
\hline$<70$ & $36(36.0 \%)$ & $12(28.6 \%)$ & $48(33.8 \%)$ & \multirow[t]{2}{*}{0.729} & 0.393 \\
\hline$>70$ & $64(64.0 \%)$ & $30(71.4 \%)$ & $94(66.2 \%)$ & & \\
\hline \multicolumn{6}{|l|}{ Gender } \\
\hline Male & 93(93.0\%) & $35(83.3 \%)$ & $128(90.1 \%)$ & \multirow[t]{2}{*}{3.110} & 0.078 \\
\hline Female & $7(7.0 \%)$ & $7(16.7 \%)$ & $14(9.9 \%)$ & & \\
\hline \multicolumn{6}{|l|}{$\begin{array}{l}\text { Tumour } \\
\text { Grade }\end{array}$} \\
\hline G1/G2 & $67(67.0 \%)$ & $22(52.4 \%)$ & $89(62.7 \%)$ & \multirow[t]{2}{*}{2.702} & 0.100 \\
\hline G3 & $33(33.0 \%)$ & $20(47.6 \%)$ & $53(37.3 \%)$ & & \\
\hline \multicolumn{6}{|l|}{ Tumour Stage } \\
\hline $\mathrm{Ta} / \mathrm{T} 1$ & $83(83.0 \%)$ & $13(31.0 \%)$ & $96(67.6 \%)$ & \multirow[t]{2}{*}{36.586} & $0.001 *$ \\
\hline $\mathrm{T} 2$ or above & $17(17.0 \%)$ & $29(69.0 \%)$ & $46(32.4 \%)$ & & \\
\hline \multicolumn{6}{|l|}{ Tumour Size } \\
\hline$<3 \mathrm{~cm}$ & $78(78.0 \%)$ & $23(54.8 \%)$ & $101(71.1 \%)$ & \multirow[t]{2}{*}{7.777} & $0.005^{*}$ \\
\hline$>3 \mathrm{~cm}$ & $22(22.0 \%)$ & $19(45.2 \%)$ & $41(28.9 \%)$ & & \\
\hline \multicolumn{6}{|l|}{$\begin{array}{c}\text { Tumour } \\
\text { Multiplicity }\end{array}$} \\
\hline Solitary & $62(62.0 \%)$ & $32(76.2 \%)$ & $94(66.2 \%)$ & \multirow[t]{2}{*}{2.662} & 0.103 \\
\hline Multiple & $38(38.0 \%)$ & $10(23.8 \%)$ & $48(33.8 \%)$ & & \\
\hline
\end{tabular}

$* \mathrm{p}<0.05$, statistically significant

Table: 3 Baseline characteristics of patients associated with Platelet-to-lymphocyte ratio (PLR)

\begin{tabular}{|c|c|c|c|c|c|}
\hline \multirow[t]{2}{*}{ Age } & \multicolumn{2}{|c|}{ PLR } & \multirow[t]{2}{*}{ Total } & \multirow[t]{2}{*}{ Chi square value } & \multirow{2}{*}{$\mathrm{p}$ value } \\
\hline & $<218$ & $>218$ & & & \\
\hline$<70$ & $39(35.1 \%)$ & $9(29.0 \%)$ & $48(33.8 \%)$ & \multirow[t]{2}{*}{0.403} & \multirow[t]{2}{*}{0.525} \\
\hline$>70$ & $72(64.9 \%)$ & $22(71.0 \%)$ & $94(66.2 \%)$ & & \\
\hline \multicolumn{6}{|l|}{ Gender } \\
\hline Male & $105(94.6 \%)$ & $23(74.2 \%)$ & $128(90.1 \%)$ & \multirow[t]{2}{*}{11.349} & \multirow[t]{2}{*}{$0.001^{\prime \prime}$} \\
\hline Female & $6(5.4 \%)$ & $8(25.8 \%)$ & $14(9.9 \%)$ & & \\
\hline \multicolumn{6}{|l|}{$\begin{array}{l}\text { Tumour } \\
\text { Grade }\end{array}$} \\
\hline G1/G2 & $72(64.9 \%)$ & $17(54.8 \%)$ & $89(62.7 \%)$ & \multirow[t]{2}{*}{1.041} & \multirow[t]{2}{*}{0.308} \\
\hline G3 & $39(35.1 \%)$ & $14(45.2 \%)$ & $53(37.3 \%)$ & & \\
\hline \multicolumn{6}{|l|}{ Tumour Stage } \\
\hline $\mathrm{Ta} / \mathrm{T} 1$ & $81(73.0 \%)$ & $15(48.4 \%)$ & $96(67.6 \%)$ & \multirow[t]{2}{*}{6.688} & \multirow[t]{2}{*}{$0.010^{*}$} \\
\hline $\mathrm{T} 2$ or above & $30(27.0 \%)$ & $16(51.6 \%)$ & $46(32.4 \%)$ & & \\
\hline \multicolumn{6}{|l|}{ Tumour Size } \\
\hline$<3 \mathrm{~cm}$ & $93(83.8 \%)$ & $8(25.8 \%)$ & $101(71.1 \%)$ & \multirow[t]{2}{*}{39.663} & \multirow[t]{2}{*}{$0.001 *$} \\
\hline$>3 \mathrm{~cm}$ & $18(16.2 \%)$ & $23(74.2 \%)$ & $41(28.9 \%)$ & & \\
\hline $\begin{array}{c}\text { Tumour } \\
\text { Multiplicity }\end{array}$ & & & & & \\
\hline Solitary & $71(64.0 \%)$ & $23(74.2 \%)$ & $94(66.2 \%)$ & 1.133 & 0.287 \\
\hline
\end{tabular}

${ }^{*} \mathrm{p}<0.05$, statistically significant 
Table: 4 Baseline characteristics of patients associated with Lymphocyte-to-monocyte ratio (LMR)

\begin{tabular}{|c|c|c|c|c|c|}
\hline \multirow[t]{2}{*}{ Age } & \multicolumn{2}{|c|}{ LMR } & \multirow[t]{2}{*}{ Total } & \multirow[t]{2}{*}{ Chi square value } & \multirow[t]{2}{*}{$\mathrm{p}$ value } \\
\hline & $<1.8$ & $>1.8$ & & & \\
\hline$<70$ & $40(35.1 \%)$ & $8(28.6 \%)$ & $48(33.8 \%)$ & \multirow[t]{2}{*}{0.427} & \multirow[t]{2}{*}{0.514} \\
\hline$>70$ & $74(64.9 \%)$ & $20(71.4 \%)$ & $94(66.2 \%)$ & & \\
\hline \multicolumn{6}{|l|}{ Gender } \\
\hline Male & $106(93.0 \%)$ & $22(78.6 \%)$ & $128(90.1 \%)$ & \multirow[t]{2}{*}{5.253} & \multirow[t]{2}{*}{$0.022 *$} \\
\hline Female & $8(7.0 \%)$ & $6(21.4 \%)$ & $14(9.9 \%)$ & & \\
\hline \multicolumn{6}{|l|}{$\begin{array}{l}\text { Tumour } \\
\text { Grade }\end{array}$} \\
\hline G1/G2 & $71(62.3 \%)$ & $18(64.3 \%)$ & $89(62.7 \%)$ & \multirow[t]{2}{*}{0.039} & \multirow[t]{2}{*}{0.844} \\
\hline G3 & $43(37.7 \%)$ & $10(35.7 \%)$ & $53(37.3 \%)$ & & \\
\hline \multicolumn{6}{|l|}{ Tumour Stage } \\
\hline $\mathrm{Ta} / \mathrm{T} 1$ & $86(75.4 \%)$ & $10(35.7 \%)$ & $96(67.6 \%)$ & \multirow[t]{2}{*}{16.197} & \multirow[t]{2}{*}{$0.001 *$} \\
\hline T2 or above & $28(24.6 \%)$ & $18(64.3 \%)$ & $46(32.4 \%)$ & & \\
\hline \multicolumn{6}{|l|}{ Tumour Size } \\
\hline$<3 \mathrm{~cm}$ & $96(84.2 \%)$ & $5(17.9 \%)$ & $101(71.1 \%)$ & \multirow[t]{2}{*}{48.192} & \multirow[t]{2}{*}{$0.001 *$} \\
\hline$>3 \mathrm{~cm}$ & $18(15.8 \%)$ & $23(82.1 \%)$ & $41(28.9 \%)$ & & \\
\hline \multicolumn{6}{|l|}{$\begin{array}{c}\text { Tumour } \\
\text { Multiplicity }\end{array}$} \\
\hline Solitary & $72(63.2 \%)$ & $22(78.6 \%)$ & $94(66.2 \%)$ & \multirow[t]{2}{*}{2.387} & \multirow[t]{2}{*}{0.122} \\
\hline Multiple & $42(36.8 \%)$ & $6(21.4 \%)$ & $48(33.8 \%)$ & & \\
\hline
\end{tabular}

$* \mathrm{p}<0.05$, statistically significant.

Table: 5 Patient characteristics, prognostic scores and tumour characteristics associated with tumour stage

\begin{tabular}{|c|c|c|c|c|c|}
\hline \multirow[t]{2}{*}{ Age } & \multicolumn{2}{|c|}{ Tumour Stage } & \multirow[t]{2}{*}{ Total } & \multirow[t]{2}{*}{ Chi square value } & \multirow[t]{2}{*}{$\mathrm{p}$ value } \\
\hline & $\mathrm{Ta} / \mathrm{T} 1$ & T2 or above & & & \\
\hline$<70$ & $35(36.5 \%)$ & $13(28.3 \%)$ & $48(33.8 \%)$ & \multirow[t]{2}{*}{0.934} & \multirow[t]{2}{*}{0.334} \\
\hline$>70$ & $61(63.5 \%)$ & $33(71.7 \%)$ & $94(66.2 \%)$ & & \\
\hline \multicolumn{6}{|l|}{ Gender } \\
\hline Male & $89(92.7 \%)$ & $39(84.8 \%)$ & $128(90.1 \%)$ & \multirow[t]{2}{*}{2.198} & \multirow[t]{2}{*}{0.138} \\
\hline Female & $7(7.3 \%)$ & $7(15.2 \%)$ & $14(9.9 \%)$ & & \\
\hline \multicolumn{6}{|l|}{ NLR } \\
\hline$<3.89$ & $83(86.5 \%)$ & $17(37.0 \%)$ & $100(70.4 \%)$ & \multirow[t]{2}{*}{36.586} & \multirow[t]{2}{*}{$0.001 *$} \\
\hline$>3.89$ & $13(13.5 \%)$ & $29(63.0 \%)$ & $42(29.6 \%)$ & & \\
\hline \multicolumn{6}{|l|}{ PLR } \\
\hline$<218$ & $81(84.4 \%)$ & $30(65.2 \%)$ & $111(78.2 \%)$ & \multirow[t]{2}{*}{6.688} & \multirow[t]{2}{*}{$0.010^{*}$} \\
\hline$>218$ & $15(15.6 \%)$ & $16(34.8 \%)$ & $31(21.8 \%)$ & & \\
\hline \multicolumn{6}{|l|}{ LMR } \\
\hline$<1.8$ & $86(89.6 \%)$ & $28(60.9 \%)$ & $114(80.3 \%)$ & \multirow[t]{2}{*}{16.197} & \multirow[t]{2}{*}{$0.001 *$} \\
\hline$>1.8$ & $10(10.4 \%)$ & $18(39.1 \%)$ & $28(19.7 \%)$ & & \\
\hline \multicolumn{6}{|c|}{ Tumour Grade } \\
\hline $\mathrm{G} 1 / \mathrm{G} 2$ & $61(63.5 \%)$ & $28(60.9 \%)$ & $89(62.7 \%)$ & 0.095 & 0.758 \\
\hline G3 & $35(36.5 \%)$ & $18(39.1 \%)$ & $53(37.3 \%)$ & & \\
\hline \multicolumn{6}{|c|}{ Tumour Size } \\
\hline$<3 \mathrm{~cm}$ & $82(85.4 \%)$ & $19(41.3 \%)$ & 101(71.1\%) & \multirow[t]{2}{*}{29.467} & \multirow[t]{2}{*}{$0.001 *$} \\
\hline$>3 \mathrm{~cm}$ & $14(14.6 \%)$ & $27(58.7 \%)$ & $41(28.9 \%)$ & & \\
\hline \multicolumn{6}{|c|}{$\begin{array}{c}\text { Tumour } \\
\text { Multiplicity }\end{array}$} \\
\hline Solitary & $57(59.4 \%)$ & $37(80.4 \%)$ & $94(66.2 \%)$ & \multirow[t]{2}{*}{6.164} & \multirow[t]{2}{*}{$0.013^{*}$} \\
\hline Multiple & $39(40.6 \%)$ & $9(19.6 \%)$ & $48(33.8 \%)$ & & \\
\hline
\end{tabular}

$* \mathrm{p}<0.05$, statistically significant. 
Table: 6 Multivariate logistic regression analysis

\begin{tabular}{|c|c|c|c|c|}
\hline \multirow[b]{2}{*}{ Variable } & \multirow{2}{*}{ Odds Ratio } & \multicolumn{2}{|c|}{ 95\% Confidence Interval } & \multirow[b]{2}{*}{$\mathrm{p}$ value } \\
\hline & & Lower bound & Upper bound & \\
\hline NLR $(\geq 3.89)$ & 11.822 & 4.492 & 31.112 & $0.001 *$ \\
\hline PLR $(\geq 218)$ & 0.633 & 0.179 & 2.244 & 0.479 \\
\hline $\operatorname{LMR}(\geq 1.8)$ & 2.229 & 0.606 & 8.197 & 0.228 \\
\hline $\begin{array}{c}\text { Tumour size } \\
(\geq 3 \mathrm{~cm}, \text { large })\end{array}$ & 6.306 & 1.563 & 25.436 & $0.010^{*}$ \\
\hline Multiplicity (multiple) & 0.379 & 0.117 & 1.228 & 0.106 \\
\hline
\end{tabular}

Binomial logistic regression analysis was performed, including prognostic scores, patient characteristics and tumour characteristics, to identify factors associated with muscle-invasive bladder cancer (MIBC). Univariate analysis identified NLR, PLR, LMR, Tumour size and Tumour multiplicity as significant predictors of muscle-invasive bladder cancer (MIBC) Table: 5. NLR (OR, 11.822; 95\% CI, 4.492 - 31.112; $\mathrm{p}=0.001)$ and tumour size $(\mathrm{OR}, 6.306 ; 95 \% \mathrm{CI}$, 1.563 - 25.436; $\mathrm{p}=0.010$ ) were identified as independent predictors of muscle-invasive bladder cancer (MIBC) by the multivariate logistic regression model

\section{Discussion}

Bladder cancer is a heterogeneous disease. The optimum management of bladder cancer is guided by accurate staging, for which pathological analysis is the gold standard. Staging error is extremely common, with $50 \%$ of patients, at time of radical cystectomy, demonstrates upstaging ${ }^{[12,13]}$. Researchers have attempted to improve the staging process by combining histology, molecular markers and imaging modalities such as computed tomography or magnetic resonance imaging into predictive nomograms. Both Karakiewicz et al. ${ }^{[14]}$ and Margel et al. ${ }^{[15]}$ presented nomograms to predict organ-confined disease before cystectomy. Margel et al. utilised molecular markers for this. To improve the accuracy of these models, novel variables, including laboratory analyses, are needed ${ }^{[16]}$.

The host inflammatory responses play a critical role in carcinogenesis, with inflammatory cells and innate immune system signalling molecules being involved in tumour progression ${ }^{[2]}$. This systemic inflammatory response leads to changes in relative levels of circulating leukocytes, providing a means to measure this response, in addition to circulating acute-phase proteins, e.g., C-reactive protein, fibrinogen, ferritin, albumin, etc. Thus, NLR, PLR, and LMR, indices that represent the systemic inflammatory response, have proven useful as potential prognostic factors in cancer ${ }^{[5,7]}$.

NLR, is an independent predictor of $\mathrm{MIBC}^{[12]}$. Tumour grade and tumour size were also found to be independent predictors as both are well documented prognostic indicators in bladder cancer $^{[17]}$. While PLR and LMR were not identified as independent predictors of $\mathrm{MIBC}^{[12]}$. Kaynar et al. ${ }^{[1]}$ undertook limited analysis of PLR, and found no significant difference between mean PLR in NMIBC and MIBC (Mann-Whitney $\mathrm{U}$ test, $\mathrm{p}=0.810$ ).

NLR was first proposed as a simple index to assess the systemic inflammatory response in critically ill patients ${ }^{[18]}$. On the basis of low cost and ease of access, given that it comprises components of the routine full blood count assay, and can easily be performed prior to flexible cystoscopy or TURBT surgery, it has the advantage over other markers of inflammation. The association between NLR and invasive disease is complex. An increased neutrophildependent inflammatory reaction and decreased lymphocyte-mediated antitumour immune response is reflected by a high $\mathrm{NLR}^{[19]}$.

Through TURBT, patients must continue to have formal pathological diagnosis of MIBC clinically. However, inability to identify all cases of MIBC is one pitfall of TURBT. As many as $48 \%$ of patients were found to harbour muscle-invasive 
disease, in studies analysing patients undergoing radical cystectomy for clinical $\mathrm{T} 1$ disease after TURBT $^{[13]}$. For accurate pathological staging, good quality tumour resections are required. Variables such as the presence of detrusor muscle in the tumour specimen can provide a proxy indicator for resection quality ${ }^{[20]}$. Patients at high risk for muscle-invasive disease may be identified using NLR as a potential marker. For example, a high NLR may guide the clinician towards planning further therapy. In these high risk patients, this could include early follow-up flexible cystoscopy or repeat TURBT to identify any residual muscle-invasive disease, missed during the first surgery. However, further evaluation is required for the use of NLR in a clinical setting.
Our results agree with previous studies. They have examined the relationship between NLR and bladder cancer staging, summarised in Table 7 . The consensus finding in these studies was elevated NLR in MIBC as compared to NMIBC. However, two research groups performed univariate analysis only ${ }^{[9,10,11]}$. Elevated NLR was associated with upstaging or extra-vesical disease in the papers examining patients after radical cystectomy, ${ }^{[21,22,23]}$. There is wide range of ROC curve cutoff values for NLR in these publications. The nature of NLR as a nonspecific marker that may rise secondary to a number of insults, such as infection may explains this variation. A consens cutoff value for NLR remains to be determined.

Table 3. Previous studies comparing NLR and bladder cancer staging

\begin{tabular}{|c|c|c|c|}
\hline Source & Patient & NLR & Analysis \\
\hline Can et al. (2012) & $\begin{array}{l}182 \text { Patients: NMIBC } \\
(\mathrm{n}=80), \text { MIBC }(\mathrm{n}=102)\end{array}$ & ROC cutoff: 2.57 & $\begin{array}{l}\text { NLR> } 2.57 \text { was independent predictor of MIBC } \\
\quad\left(\mathrm{OR}, 2.78 ; 95 \% \mathrm{CI}, 1.383-5.588 ; \mathrm{p}=0.004^{*}\right)\end{array}$ \\
\hline $\begin{array}{l}\text { Ceylan et al. } \\
(2014)^{[10]}\end{array}$ & $\begin{array}{l}198 \text { Patients: NMIBC } \\
(n=162), \text { MIBC }(n=36)\end{array}$ & $\begin{array}{c}\text { Mean NLR: MIBC, } \\
4.14 \pm 2.76 \text {; NMIBC, } 3.36 \pm 2.88 \\
\text { ROC cutoff: } 3.96\end{array}$ & $\begin{array}{l}\text { Mean NLR differed significantly between } \\
\text { MIBC and NMIBC on Mann-Whitney U test } \\
\qquad\left(\mathrm{p}=0.03^{*}\right)\end{array}$ \\
\hline $\begin{array}{l}\text { Kaynar et al. } \\
(2014)^{[11]}\end{array}$ & $\begin{array}{l}291 \text { Patients: NMIBC } \\
(\mathrm{n}=192), \text { MIBC }(\mathrm{n}=99)\end{array}$ & $\begin{array}{l}\text { Mean NLR: MIBC, } 2.9 \pm 0.2 \\
\text { NMIBC, } 2.4 \pm 0.1\end{array}$ & $\begin{array}{l}\text { Mean NLR differed significantly between } \\
\text { MIBC and NMIBC on Mann-Whitney U test } \\
\left(\mathrm{p}=0.028^{*}\right)\end{array}$ \\
\hline & & & $\begin{array}{l}\text { Significant correlation between NLR and MIBC } \\
\text { on univariate analysis }\left(\mathrm{r}=0.138, \mathrm{p}=0.031^{*}\right)\end{array}$ \\
\hline \multirow[t]{2}{*}{ Krane et al. (2013) } & $\begin{array}{l}68 \text { Patients with recurrent } \mathrm{T} 1 \\
\text { disease or MIBC undergoing } \\
\text { radical cystectomy }\end{array}$ & Mean NLR: 4.0 \pm 2.8 (overall) & $\begin{array}{c}\text { NLR }>2.5 \text { was independent predictor of } \\
\text { extravesical disease (RR, 3.18; } 95 \% \text { CI, 1.09- } \\
\text { 9.79; no p-value given) }\end{array}$ \\
\hline & & $\begin{array}{c}\text { Cutoff: } 2.5 \text { (as per previous } \\
\text { publications) }\end{array}$ & \\
\hline $\begin{array}{l}\text { Potretzke et al. } \\
\quad(2014)^{[23]}\end{array}$ & $\begin{array}{l}102 \text { Patients undergoing } \\
\text { radical cystectomy: NMIBC } \\
(\mathrm{n}=25), \operatorname{MIBC}(\mathrm{n}=77)\end{array}$ & $\begin{array}{l}\text { Mean NLR: } 4.33 \pm 0.87 \\
\text { (upstaged to } \geq \mathrm{pT} 3) \text { and } \\
2.66 \pm 0.29(\leq \mathrm{pT} 2)\end{array}$ & $\begin{array}{c}\text { NLR (continuous variable) was independent } \\
\text { predictor of upstaging }(\mathrm{OR}, 1.36 ; 95 \% \mathrm{CI}, 1.01 \text { - } \\
\left.1.84 ; \mathrm{p}=0.04^{*}\right) \text { and extravesical dis- ease }(\mathrm{OR}, \\
\left.1.5 ; 95 \% \mathrm{CI}, 1.07-2.1 ; \mathrm{p}=0.02^{*}\right) \text { at radical } \\
\text { cystectomy }\end{array}$ \\
\hline Viers et al. (2014) & $\begin{array}{l}899 \text { Patients undergoing } \\
\text { radical cystectomy: NMIBC } \\
(\mathrm{n}=363), \operatorname{MIBC}(\mathrm{n}=524)\end{array}$ & Cutoff: 2.7 (obtained visually) & $\begin{array}{l}\text { NLR (continuous variable) was independent } \\
\text { predictor of extravesical disease (OR, } 1.07 \text {; } \\
\left.\text { 95\% CI, } 1.01-1.15 ; \mathrm{p}=0.03^{*}\right) \text { and lymph node } \\
\text { involvement }(\mathrm{OR}, 1.09 ; 95 \% \mathrm{CI}, 1.02-1.16 \\
\left.\mathrm{p}=0.02^{*}\right)\end{array}$ \\
\hline 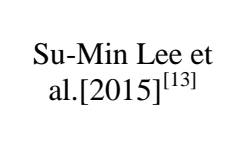 & $\begin{array}{l}226 \text { Patients undergoing } \\
\text { TURBT: NMIBC }(\mathrm{n}=175) \\
\text { MIBC }(\mathrm{n}=51)\end{array}$ & NLR cutoff 3.89 & $\begin{array}{c}\text { NLR (threshold, >3.89) had the greatest area } \\
\text { under the ROC curve (AUC) of } 0.710 \\
\text { (sensitivity, 52.9\%; specificity, } 84.0 \% \\
\text { p <0.0001) }\end{array}$ \\
\hline
\end{tabular}

NLR, neutrophil-to-lymphocyte ratio; NMIBC, nonmuscle invasive bladder cancer; MIBC, muscle invasive bladder cancer; ROC, receiver operating characteristics; OR, odds ratio; CI, confidence interval; RR, relative risk.

Many authors have found patients with elevated NLR as an independent predictor for recurrencefree, disease-specific and overall survival ${ }^{[21,23]}$.
Mano et al. ${ }^{[24]}$ examined a group of NMIBC patients and found an elevated NLR to be significantly associated with disease progression 
and recurrence. Ozyalvacli et al. ${ }^{[25]}$ further limited their patient cohort to those with stage pT1 bladder tumours, and confirmed the association of NLR with disease recurrence and progression. This association of elevated NLR with disease recurrence, invasive disease and survival could signify a marker for a subset of patients with high risk, aggressive tumour biology.

We recognise the limitations of our study. While we omitted patients with concurrent inflammatory conditions (e.g., infection, haematological disorder), the confounding effect of these cannot be completely excluded.

Furthermore, the main aim of our study was to differentiate muscle-invasive from superficial disease. The groups were separated into NMIBC $(\mathrm{Ta} / \mathrm{T} 1)$ and MIBC $(\mathrm{T} 2+)$. These differentiated patients who would be appropriately managed with TURBT as compared to radical surgery, radiotherapy or chemotherapy, as per current European Association of Urology guidelines [26]. The muscle-invasive, or $\mathrm{T} 2+$ disease does include a wide range of disease. This can include cancer that has invaded the muscularis propria (T2), up to adjacent structures (T4), such as the prostate, vagina or pelvic wall. So it is likely that the systemic inflammatory response increases with tumour stage. The use of inflammation-based prognostic scoring to differentiate each stage of MIBC was beyond the scope of our study. It merits further study to identify patients most appropriate for radical surgery.

Despite these limitations, NLR and tumour size appears to be a promising marker for invasive bladder cancer and may be a useful variable in future predictive nomograms. To fully define the utility of NLR within a clinical setting, larger, prospective studies are required

\section{Conclusion}

Accurate pathological staging in bladder cancer is vital. This will guide proper and early management. Our comparison of pre treatment inflammation-based prognostic scores indicates that NLR and tumour size are independent predictors of muscle-invasive disease. NLR may provide a simple, cost-effective and easily measured marker for MIBC. It can be performed at the time of diagnostic flexible cystoscopy, thereby assisting in the planning of further treatment and follow-up.

Our sincere thanks to Sony Simon for her assistance in statistical analysis

\section{References}

1. Cancer statistics, 2016.Siegel RL, Miller KD, Jemal A. CA Cancer J Clin. 2016 JanFeb;66(1):7-30. doi: 10.3322/caac.21332. Epub 2016 Jan 7. PMID:2674299

2. Coussens LM, Werb Z. Inflammation and cancer. Nature 2002;420:860-867.

3. EAU guidelines on non-muscle-invasive urothelial carcinoma of the bladder: Babjuk M, Burger M, Zigeuner R, Shariat $\mathrm{SF}$, van Rhijn BW, Comperat E, et al. update 2013. Eur Urol 2013;64:639-653.

4. Dutta SC, Smith JA Jr, Shappell SB, Coffey CS, Chang SS, Cookson MS. Clinical under staging of high risk nonmuscle invasive urothelial carcinoma treated with radical cystectomy. J Urol 2001;166:490-493.

5. Kumar R, Geuna E, Michalarea V, Guardascione M, Naumann U, Lorente D, et al. The neutrophil-lymphocyte ratio and its utilisation for the management of cancer patients in early clinical trials. $\mathrm{Br} \mathbf{J}$ Cancer 2015;112:1157-1165.

6. Proctor MJ, McMillan DC, Morrison DS, Fletcher CD, Horgan PG, Clarke SJ. A derived neutrophil to lymphocyte ratio predicts survival in patients with cancer. Br J Cancer 2012;107:695-699.

7. Roxburgh CS, McMillan DC. Role of systemic inflammatory response in predicting survival in patients with primary operable cancer. Future Oncol 2010;6:149-163.

8. Zhou X, Du Y, Huang Z, Xu J, Qiu T, Wang J, et al. Prognostic value of PLR in 
various cancers: a meta-analysis. PLoS One 2014;9:e101119.

9. Can C, Baseskioglu B, Yilmaz M, Colak E, Ozen A, Yenilmez A. Pretreatment parameters obtained from peripheral blood sample predicts invasiveness of bladder carcinoma. Urol Int 2012;89:468-472.

10. Ceylan C, Doluoglu OG, Keles I, Gazel E, Temucin T, Odabas O, et al. Importance of the neutrophil-to-lymphocyte ratio in muscle-invasive and non-muscle invasive bladder tumors. Urologia 2014;81:120124.

11. Kaynar M, Yıldırım ME, Badem H, Cavis M, Tekinarslan E, Istanbulluoglu MO, et al. Bladder cancer invasion predictability based on preoperative neutrophillymphocyte ratio. Tumour Biol 2014;35: 6601-6605.

12. Su-Min Lee, Andrew Russell, and Giles Hellawell ,Korean J Urol. 2015 Nov;56(11):749-755. Predictive value of pretreatment inflammation-based prognostic scores for invasive bladder carcinoma.

13. Shariat SF, Palapattu GS, Karakiewicz PI, Rogers CG, Vazina A, Bastian PJ, et al. Discrepancy between clinical and pathologic stage: impact on prognosis after radical cystectomy. Eur Urol 2007;51:137-149.

14. Ark JT, Keegan KA, Barocas DA, Morgan TM, Resnick MJ, You C, et al. Incidence and predictors of understaging in patients with clinical T1 urothelial carcinoma undergoing radical cystectomy. BJU Int 2014;113:894-899.

15. Karakiewicz PI, Shariat SF, Palapattu GS, Gilad AE, Lotan Y, Rogers CG, et al. Nomogram for predicting disease recurrence after radical cystectomy for transitional cell carcinoma of the bladder. $\mathbf{J}$ Urol 2006;176(4 Pt 1):1354-1361.

16. Margel D, Harel A, Yossepowitch O, Baniel J. A novel algorithm to improve pathologic stage prediction of clinically organ-confined muscle-invasive bladder cancer. Cancer 2009;115:1459-1464.

17. Bostrom PJ, Van Rhijn BW, Fleshner N, Finelli A, Jewett M, Thoms J, et al. Staging and staging errors in bladder cancer. Eur Urol Suppl 2010;9:2-9.

18. Sylvester RJ, van der Meijden AP, Oosterlinck W, Witjes JA, Bouffioux C, Denis L, et al. Predicting recurrence and progression in individual patients with stage Ta T1 bladder cancer using EORTC risk tables: a combined analysis of 2596 patients from seven EORTC trials. Eur Urol 2006;49:466-465.

19. Zahorec R. Ratio of neutrophil to lymphocyte counts: rapid and simple parameter of systemic inflammation and stress in critically ill. Bratisl Lek Listy 2001;102:5-14.

20. Paramanathan A, Saxena A, Morris DL. A systematic review and meta-analysis on the impact of pre-operative neutrophil lymphocyte ratio on long term outcomes after curative intent resection of solid tumours. Surg Oncol 2014;23:31-39.

21. Mariappan P, Finney SM, Head E, Somani BK, Zachou A, Smith G, et al. Good quality white-light transurethral resection of bladder tumours (GQ-WLTURBT) with experienced surgeons performing complete resections and obtaining detrusor muscle reduces early recurrence in new nonmuscle-invasive bladder cancer: validation across time and place and recommendation for benchmarking. BJU Int 2012;109: 1666-1673.

22. Krane LS, Richards KA, Kader AK, Davis R, Balaji KC, Hemal AK. Preoperative neutrophil/lymphocyte ratio predicts overall survival and extravesical disease in patients undergoing radical cystectomy. J Endourol 2013;27:1046-1050.

23. Potretzke A, Hillman L, Wong K, Shi F, Brower R, Mai $S$, et al. NLR is predictive 
of upstaging at the time of radical cystectomy for patients with urothelial carcinoma of the bladder. Urol Oncol 2014;32:631-636.

24. Viers BR, Boorjian SA, Frank I, Tarrell RF, Thapa P, Karnes RJ, et al. Pretreatment neutrophil-to-lymphocyte ratio is associated with advanced pathologic tumor stage and increased cancer-specific mortality among patients with urothelial carcinoma of the bladder undergoing radical cystectomy. Eur Urol 2014;66:1157-1164.

25. Mano R, Baniel J, Shoshany O, Margel D, Bar-On T, Nativ O, et al. Neutrophil-tolymphocyte ratio predicts progression and recurrence of non-muscle-invasive bladder cancer. Urol Oncol 2015;33:67.e1-67.e7.

26. Ozyalvacli ME, Ozyalvacli G, Kocaaslan R, Cecen K, Uyeturk U, Kemahlı E, et al. Neutrophil-lymphocyte ratio as a predictor of recurrence and progression in patients with high-grade pT1 bladder cancer. Can Urol Assoc J 2015;9:E126-E131.

27. Witjes JA, Comperat E, Cowan NC, De Santis M, Gakis G, Lebret T, et al. EAU guidelines on muscle-invasive and metastatic bladder cancer: summary of the 2013 guidelines. Eur Urol 2014;65:778792. 